\title{
A novel feature extraction method based on highly expressed SNPs for tissue-specific gene prediction
}

\author{
Jasbir Dhaliwal ${ }^{1 *}$ (1) and John Wagner ${ }^{2}$
}

\author{
*Correspondence: \\ jasbirkaur.dhaliwal@monash. \\ edu \\ ${ }^{1}$ School of Information \\ Technology, Monash \\ University Malaysia, Subang \\ Jaya 47500, Malaysia \\ Full list of author information \\ is available at the end of the \\ article
}

\begin{abstract}
Background: Gene expression provides a means for an organism to produce gene products necessary for the organism to live. Variation in the significant gene expression levels can distinguish the gene and the tissue in which the gene is expressed. Tissue-specific gene expression, often determined by single nucleotide polymorphisms (SNPs), provides potential molecular markers or therapeutic targets for disease progression. Therefore, SNPs are good candidates for identifying disease progression. The current bioinformatics literature uses gene network modeling to summarize complex interactions between transcription factors, genes, and gene products. Here, our focus is on the SNPs' impact on tissue-specific gene expression levels. To the best of our knowledge, we are not aware of any studies that distinguish tissue-specific genes using SNP expression levels.
\end{abstract}

Method: We propose a novel feature extraction method based on highly expressed SNPs using k-mers as features. We also propose optimal k-mer and feature sizes used in our approach. Determining the optimal sizes is still an open research question as it depends on the dataset and purpose of the analysis. Therefore, we evaluate our algorithm's performance on a range of $k$-mer and feature sizes using a multinomial naive Bayes (MNB) classifier on genes in the 49 human tissues from the Genotype-Tissue Expression (GTEx) portal.

Conclusions: Our approach achieves practical performance results with k-mers of size 3. Based on the purpose of the analysis and the number of tissue-specific genes under study, feature sizes $[7,8,9]$ and $[8,9,10]$ are typically optimal for the machine learning model.

Keywords: Tissue-specific gene prediction, SNP expression level, Machine learning

\section{Background}

The Human Genome Project [1] and the International HapMap Project [2] have been widely considered as the scientific foundation for precision medicine [3]. Precision medicine uses an individual's genetic makeup to determine how a doctor can tailor a patient's therapy. An individual's genetic makeup refers to the genotype of that individual, i.e., the complete set of genes, and in a narrower sense, refers to the alleles or variant forms of a gene. Thus, it is becoming critical to understand the genetic basis of common diseases,

(c) The Author(s), 2021. Open Access This article is licensed under a Creative Commons Attribution 4.0 International License, which permits use, sharing, adaptation, distribution and reproduction in any medium or format, as long as you give appropriate credit to the original author(s) and the source, provide a link to the Creative Commons licence, and indicate if changes were made. The images or other third party material in this article are included in the article's Creative Commons licence, unless indicated otherwise in a credit line to the material. If material is not included in the article's Creative Commons licence and your intended use is not permitted by statutory regulation or exceeds the permitted use, you will need to obtain permission directly from the copyright holder. To view a copy of this licence, visit http:// creativecommons.org/licenses/by/4.0/. 
such as which genes predispose an individual to disease and rare genetic variants that can alter gene function. Moreover, SNP expression levels are powerful indicators that can be used to distinguish genes across tissues. Many studies have associated variants such as SNPs with disease progression, e.g., cardiovascular risks [4], obesity [5], hypoxia (a condition characterized by a limited oxygen supply) [6, 7], and coronary artery disease [8]. Furthermore, most existing work has focused on developing disease prediction models based on SNPs associated with a single disease only, for example, breast cancer [9], inflammatory bowel disease [10], and obesity [11]. These studies use each SNP as a feature. A small number of SNPs are used as they are associated with a single disease. This is possible as a machine learning model derives its power from its ability to differentiate patterns from the data itself. Reference [12] is a recent survey that provides a detailed review of the different supervised machine learning algorithms for disease prediction.

However, we are not aware of any study that leverages the power of machine learning to identify patterns from a much larger number of SNPs, i.e., SNPs associated with multiple diseases, and then uses them to predict their respective genes. In particular, in this study, we focus on using patterns obtained from expression levels of SNPs in each gene of human tissue and uses them to predict their respective genes. Gene expression provides a means for an organism to produce gene products necessary for the organism to live. Variation in the significant gene expression levels can distinguish the gene and the tissue in which the gene is expressed. Tissue-specific gene expression, often determined by single nucleotide polymorphisms (SNPs), provides potential molecular markers or therapeutic targets for disease progression [13]. Therefore, SNPs are good candidates for identifying disease progression.

The GTEx portal provides essential data that can be used for machine learning algorithms to mine valuable patterns from tissue-specific genes. The GTEx consortium is a community of more than a hundred researchers from various groups and countries working together to increase our understanding of how changes in genes affect human health and disease. This knowledge will improve health care for future generations [14]. In particular, the Common Fund started the GTEx Program in 2010. The GTEx portal that provides access to GTEx resources was launched in 2013 [15], and is still active. The GTEx portal catalogs genetic variation, including SNPs, to gene expression levels. Each gene has a set of SNPs associated with different expression levels, including p-values indicating the degree of significance. The lower the p-value, the more significant the SNP is associated with the gene. Currently, GTEx contains data on 49 different types of human tissues. Manual processing can be used to access the SNPs per gene or even SNPs for a group of genes. This becomes a labor-intensive task if many SNPs or even genes need to be analyzed.

The current bioinformatics literature uses gene regulatory network modeling to summarize complex interactions between transcription factors, genes, and gene products [16]. In our work, we focus solely on SNP expression levels for gene prediction and not other interacting factors such as transcription factors. Thus, we cannot use gene regulatory network modeling to study the effects of the expression levels. In contrast to existing machine learning approaches that use each SNP as a feature for single disease prediction, we focus on tissue-specific gene expression across all 49 human tissues available in the GTEx portal. Tissue-specific gene expression may also be related to multiple 
diseases, resulting in a large number of SNPs overlapping among tissues. For example, SNP rs142433332 from the DARS2 gene may be related to leukoencephalopathy with brain stem and spinal cord involvement and lactate elevation; gait imbalance; and hypertensive disorder [17]. Moreover, single disease studies use multiple samples per gene due to the small number of candidate genes. On the other hand, it is impossible to have multiple samples per gene in our study as it is too costly. More specifically, our work involves 305,572 tissue-specific genes.

Our initial exploration using each SNP as a feature for machine learning tissue-specific genes confirms the challenge presented by such a large number of genes. As expected, the machine learning model cannot be used for gene prediction as it produces very low accuracy (less than 1\% for 1925 genes of the Brain amygdala tissue). To overcome this challenge, we implement natural language processing (NLP) techniques in machine learning to predict tissue-specific genes using their highly expressed SNPs. The most critical task in any NLP application is word segmentation [18]. Nevertheless, it is challenging, especially for text without explicit word boundary delimiters, such as Chinese, Japanese, or even DNA/protein sequences. Moreover, even for space-delimited text like English, relying on white space alone does not generally result in adequate segmentation [19].

Neural networks and many machine learning algorithms cannot handle non-numerical data $[20,21]$. Thus, word embeddings, a technique that maps words from a vocabulary into a vector of real numbers, are used [22]. The most common word embedding technique is k-mer counting [20, 23-25] due to its simplicity and efficiency, where low memory is used as long as the k-mer size $k$ is small. In this technique, sample data is transformed into a sequence of $\mathrm{k}$-mer words stored in a one-dimensional numerical vector [20]. A dictionary of k-mer words is then used to count the number of times each k-mer word occurs in the sample. The studies in [20, 23-25] use 5-mers on DNA barcode, ribosomal, splice, and promoter datasets. In contrast, the study in [26] proposes trimer usage while exploring DNA sequences wrapping around histone proteins in yeast datasets [27] as well as splice and promoter datasets. On the other hand, reference [28] is a survey of current gene prediction tools and recommends using hexamers, as were shown in 1992 [29] to be the most discriminative size in identifying protein-coding genes in DNA sequences.

Another important factor related to k-mer size is the number of k-mers to be used as a feature, i.e., the feature size. The feature size should be discriminating enough to be a feature, i.e., the degree of similarities between features of other genes must be low. Thus, determining the optimal feature size is also essential for this study.

Thus, we conclude from the above studies that determining optimal k-mer and feature sizes is still an open research question, with the answer depending on the dataset and purpose of the analysis. An optimal solution for one dataset or application is not necessarily optimal for another dataset or application. The contributions of this paper include:

- Proposes a novel feature extraction algorithm based on highly expressed SNPs to select k-mers as features of varying sizes. This is because it becomes computationally expensive for a machine learning model to learn from a large number of features and genes. 
- Proposes optimal k-mer and feature sizes for tissue-specific gene prediction.

- Provides a comprehensive analysis of our experimental results on the GTEx tissuespecific datasets. We show that patterns learned from SNP expression levels with the highest and lowest p-values contain similar discriminatory power.

\section{Method}

This section presents the datasets we analyze and the necessary preprocessing, feature extraction methods, classification, and evaluation metrics.

\section{Datasets}

To test the effectiveness of our approach, we downloaded all 49 tissues from the GTEx portal [30] and selected genes with at least 25 SNPs. Table 1 summarizes the tissue and the number of selected genes in each tissue. For example, thyroid tissue has the most genes $(12,805)$, while the kidney cortex tissue has the least genes $(615)$.

\section{Feature-size-based extraction method}

If we take each k-mer as a feature, we generate a large number of features that make learning not only challenging but also computationally expensive. To perform

Table 1 The 49 human tissues and their tissue-specific genes with at least 25 SNPs. A total of 305,572 genes is listed

\begin{tabular}{|c|c|c|c|c|c|}
\hline No. & Tissue & Gene & No. & Tissue & Gene \\
\hline 1 & Adipose subcutaneous & 10,884 & 26 & Esophagus mucosa & 10,143 \\
\hline 2 & Adipose visceral omentum & 8295 & 27 & Esophagus muscularis & 9862 \\
\hline 3 & Adrenal gland & 4878 & 28 & Heart atrial appendage & 7142 \\
\hline 4 & Artery aorta & 8533 & 29 & Heart left ventricle & 6286 \\
\hline 5 & Artery coronary & 3625 & 30 & Kidney cortex & 615 \\
\hline 6 & Artery tibial & 10,730 & 31 & Liver & 3287 \\
\hline 7 & Brain amygdala & 1925 & 32 & Lung & 9536 \\
\hline 8 & Brain anterior cingulate cortex & 2961 & 33 & Minor salivary gland & 2418 \\
\hline 9 & Brain caudate basal ganglia & 4779 & 34 & Muscle skeletal & 9467 \\
\hline 10 & Brain cerebellar hemisphere & 5947 & 35 & Nerve tibial & 12,662 \\
\hline 11 & Brain cerebellum & 7204 & 36 & Ovary & 3105 \\
\hline 12 & Brain cortex & 5418 & 37 & Pancreas & 6275 \\
\hline 13 & Brain frontal cortex & 4179 & 38 & Pituitary & 5692 \\
\hline 14 & Brain hippocampus & 3007 & 39 & Prostate & 4146 \\
\hline 15 & Brain hypothalamus & 3050 & 40 & Skin not sun exposed suprapubic & 10,416 \\
\hline 16 & Brain nucleus accumbens basal ganglia & 4722 & 41 & Skin sun exposed lower leg & 11,829 \\
\hline 17 & Brain putamen basal ganglia & 3877 & 42 & Small intestine terminal ileum & 3481 \\
\hline 18 & Brain spinal cord cervical & 2390 & 43 & Spleen & 6394 \\
\hline 19 & Brain substantia nigra & 1646 & 44 & Stomach & 5344 \\
\hline 20 & Breast mammary & 7003 & 45 & Testis & 12,612 \\
\hline 21 & Cells cultured fibroblasts & 10,845 & 46 & Thyroid & 12,805 \\
\hline 22 & Cells EBV transformed lymphocytes & 2551 & 47 & Uterus & 1817 \\
\hline 23 & Colon sigmoid & 6688 & 48 & Vagina & 1816 \\
\hline 24 & Colon transverse & 7417 & 49 & Whole blood & 8940 \\
\hline 25 & Esophagus gastroesophageal junction & 6928 & & & \\
\hline
\end{tabular}


dimensionality reduction on the set of k-mers, we propose using the number of k-mers in a particular range, which we call feature size, as shown in Fig. 1. The example shown in this figure demonstrates feature creation for the TSPAN6 gene indicated by the GENCODE Gene ID, i.e., ENSG00000000003.14. First, all of the SNPs indicated by the 'alt' field are collected. Then, the SNPs are sorted based on the expression levels, indicated by the 'pval_nominal' field, before being concatenated into a string. Finally, features are created based on feature size.

While exploring several $\mathrm{k}$-mer and feature sizes, we found that using three subsequent numbers as partition sizes gives enough discriminating power during the classification task. For example, the first feature consists of seven k-mers, i.e., "CCT CTG TGG GGG GGC GCA CAC", the second feature consists of eight k-mers, i.e., "CCT CTG TGG GGG GGC GCA CAC ACC", while the last feature consists of nine k-mers, i.e., "CCT CTG TGG GGG GGC GCA CAC ACC CCC". Both the k-mer and feature sizes parameters can either underfit or overfit the machine learning model. Thus, the effects of varying these parameters are explored in the experiments.

The pseudocode of the feature-size-based feature extraction method is presented as Algorithm 1. Pseudocode FSE begins by reading all the SNPs per gene and then sorting them based on the lowest p-value to extract the top $x$ highly expressed SNPs. (During our initial explorations, we were not sure on the exact number of SNPs to use when

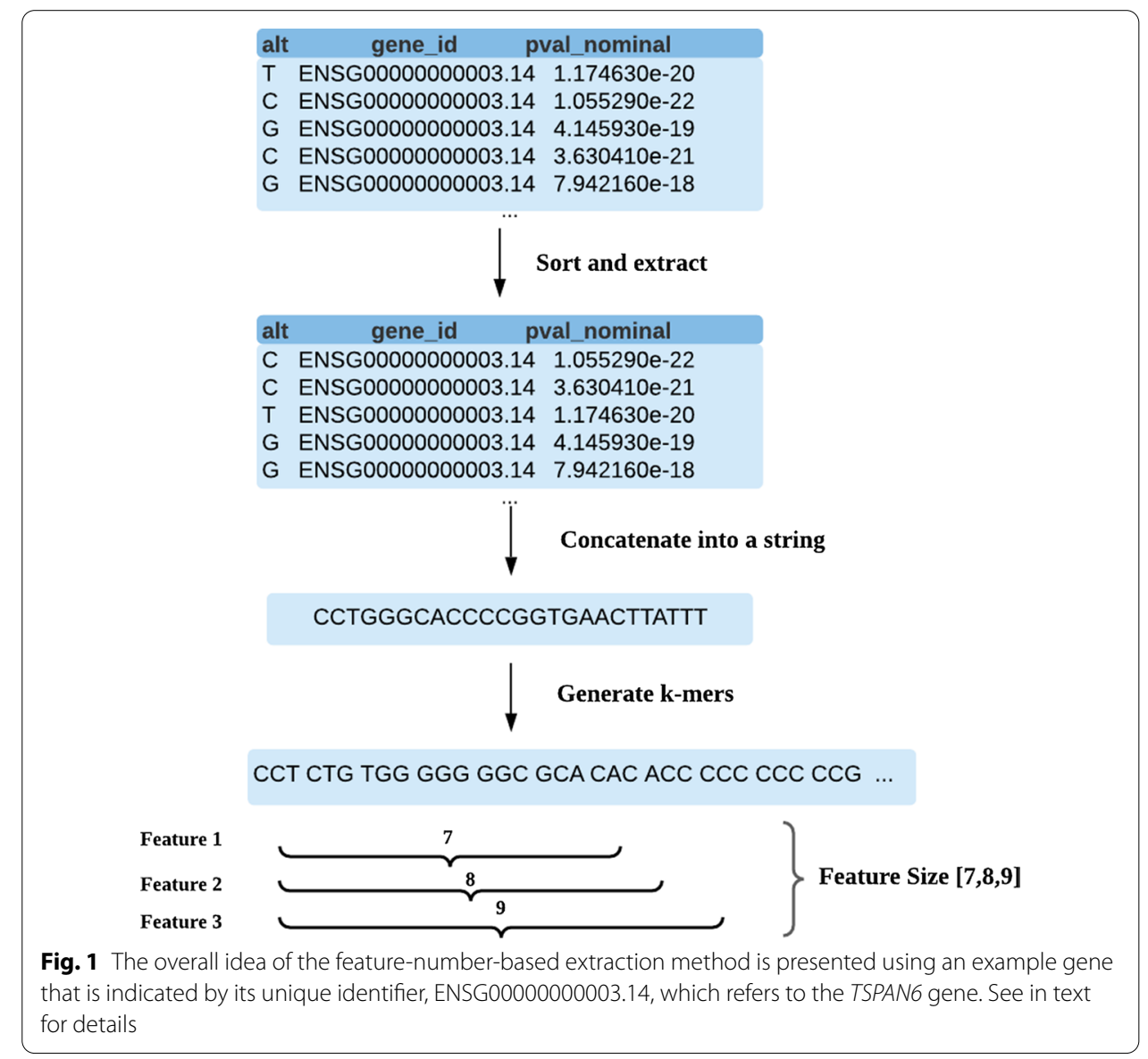


selecting genes. Taking a larger number, such as 100 reduces the number of genes per tissue that are selected for our experimental datasets. Taking $x=25$ yielded most genes in all tissues.) These SNPs are concatenated into a string. A sequence of $\mathrm{k}$-mers is then generated, where the feature size, $[a, b, c]$, is used to create a feature set, consisting of features of lengths $a, b$, and $c$, which we call the forward feature set. In contrast, the reverse feature set is created by sorting based on the highest $\mathrm{p}$-value. Note that, because the largest feature size considered in our work is $[10,11,12]$, we only need the $14(c+2$, where $c=12$ ) most highly expressed SNPs for feature creation. Source code is attached as additional supplementary material (Additional file 1: Code S1).

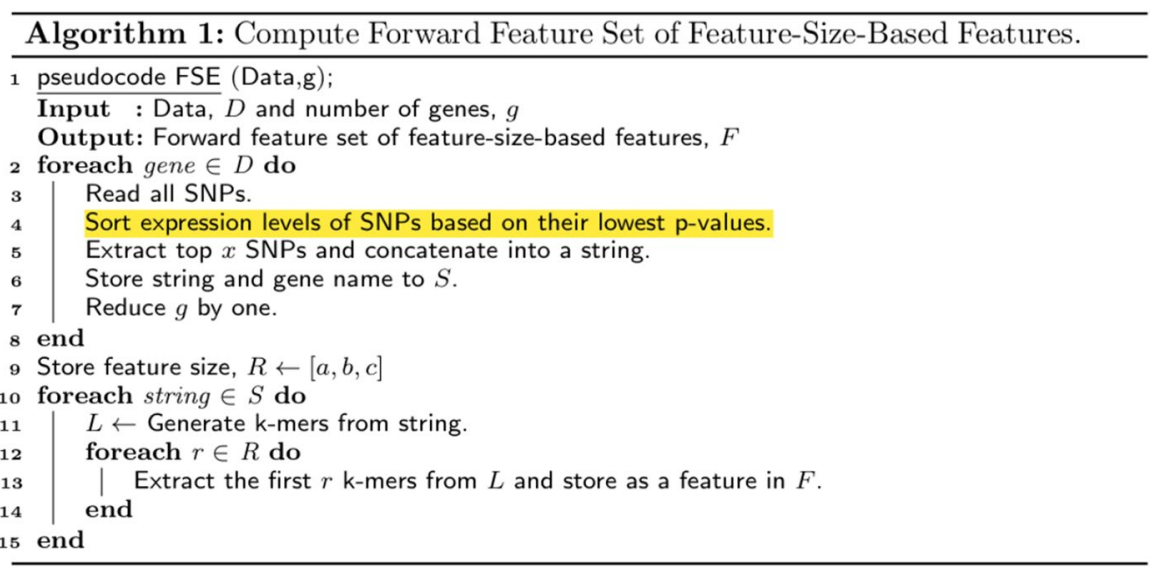

\section{Classification}

We chose the multinomial naive Bayes (MNB) classifier due to its computational efficiency and optimality for classification tasks even when the conditional independence assumption is invalid [31,32]. Moreover, it is known to outperform even more sophisticated classification methods, such as decision trees and k-nearest neighbors [33].

For the first experiment, we used forward and reverse feature sets on the MNB classifier on two tissues: Adipose subcutaneous, representing a large dataset (10,884 genes), which we refer to as Test-1; and a small dataset, Brain amygdala (1925 genes), which we refer to as Test-2. These tissues are also chosen because they are quite distinct in that one represents adipose subcutaneous samples from the leg while the other represents neural tissues sampled from a structure deep within the brain. The purpose of this experiment was twofold. First, it determines the top $x$ number of SNPs required for the gene prediction. Second, it determines the optimal k-mer and feature sizes without underfitting or overfitting the machine learning model.

To further gauge the performance of the selected k-mer and feature sizes, we tested the machine learning model on all 49 human tissues shown in Table 1 , which we refer to as Test-3, as a second experiment. Finally, the last experiment explores the efficacy of the $\mathrm{k}$-mer and feature sizes on the 175 genes common to all tissues, resulting in a dataset comprising 8575 genes, which we refer to as Test-4. This experiment is different from the other two experiments as it shows the discriminative power of SNPs expression levels to 
identify genes across different tissues. In other words, even though different tissues may express the same gene, the SNP expression level of the gene may be different and may be used to classify the tissues. These distinguishing genes are important as they are also potential biomarkers related to a disease or tissue.

\section{Evaluation metrics}

We evaluated our experiments using two machine learning evaluation metrics: accuracy and F1-score. All values were between 0 and 1. Accuracy is a metric tied to precision and recall. High precision and recall scores show that the classifier gives accurate results (high precision), and the majority of the results are positive (recall). The F1-score considers both precision and recall by taking their harmonic mean. A micro-average considers each sample equally, whereas a macro-average considers each class equally. The former is preferable for imbalanced datasets, and the latter is preferable for balanced datasets. Both the micro- and macro-averages will report the same scores if the datasets are balanced. This paper reports the macro-average F1-scores as each gene has the same number of features.

Machine learning models automatically learn patterns from a certain amount of training and may adapt too much to the dataset's peculiarities during training. Thus, it is crucial to evaluate the model on a dataset that is unseen during training. However, the common approach of dividing the dataset into separate training and test subsets does not make the most efficient use of data. An alternative is the stratified k-fold cross-validation technique. This technique ensures that each fold is a good representative of each class by splitting the dataset into $\mathrm{k}$ equal parts known as folds, and $\mathrm{k}$ training/test cycles are performed. For each cycle, a fold is set aside for testing, and the remaining k-1 folds are used for training. The reported evaluation score is the average of scores obtained during each cycle. All data is eventually used for both training and testing, but each test set's data is never accessible to the model during training for that test set [34]. Thus, the feature extraction algorithm was evaluated using stratified-3-fold cross-validation as we have three features per gene. Furthermore, we evaluated the efficiency of our method in time-space tradeoff using total run time and peak RAM usage.

\section{Results and discussion}

Experiments were conducted on the Test-1, Test-2, Test-3, and Test- 4 datasets on an otherwise ideal 3.1 GHz Dual-Core Intel Core i5 with 16 GB main memory. The operating system was macOS Big Sur.

The Test- 1 dataset comprises 10,884 genes. Both the forward and reverse feature sets consisting of 32,652 features each, used about the same run time ( $<2$ minutes) and peak memory $(<2.2 \mathrm{~GB})$ with varying k-mer and feature sizes. Generally, underfitting occurs with a k-mer size of 2 , and overfitting occurs with k-mer sizes of 4 and 5 . The fit is optimal with a k-mer size of 3 . In regards to feature sizes, underfitting occurs with feature sizes [7, 8, 9] and below. In contrast, overfitting occurs with feature size [10, 11, 12], and possibly $[9,10,11]$. The fit is always optimal with feature size $[8,9,10]$. See Table $2 a$ for details.

Likewise, in Table $2 \mathrm{~b}$, we show the same measures for the Test-2 dataset, consisting of 1925 genes. The forward and reverse feature sets comprising 5,775 features each, used 
Table 2 Effect of varying k-mer and feature sizes on the Adipose subcutaneous tissue representing the Test- 1 dataset and the Brain amygdala tissue representing the Test- 2 dataset

\begin{tabular}{|c|c|c|c|c|c|c|c|c|c|}
\hline \multirow[t]{3}{*}{ k-mer size } & \multirow[t]{3}{*}{ Feature size } & \multicolumn{8}{|c|}{ Feature set } \\
\hline & & \multicolumn{4}{|c|}{ Forward } & \multicolumn{4}{|c|}{ Reverse } \\
\hline & & Acc & F1 & Time & Mem & Acc & F1 & Time & Mem \\
\hline \multirow[t]{5}{*}{2} & {$[6,7,8]$} & 0.25 & 0.19 & 104 & 1906 & 0.26 & 0.20 & 105 & 1905 \\
\hline & {$[7,8,9]$} & 0.38 & 0.31 & 102 & 1907 & 0.39 & 0.32 & 102 & 1907 \\
\hline & {$[8,9,10]$} & 0.51 & 0.44 & 101 & 1908 & 0.51 & 0.44 & 104 & 1907 \\
\hline & {$[9,10,11]$} & 0.61 & 0.54 & 109 & 1908 & 0.61 & 0.54 & 102 & 1906 \\
\hline & {$[10,11,12]$} & 0.68 & 0.62 & 112 & 1907 & 0.68 & 0.61 & 106 & 1906 \\
\hline \multirow[t]{5}{*}{3} & {$[6,7,8]$} & 0.84 & 0.80 & 103 & 1923 & 0.85 & 0.82 & 104 & 1924 \\
\hline & {$[7,8,9]$} & 0.93 & 0.91 & 103 & 1924 & 0.94 & 0.93 & 105 & 1924 \\
\hline & {$[8,9,10]$} & 0.97 & 0.97 & 104 & 1924 & 0.98 & 0.97 & 102 & 1924 \\
\hline & {$[9,10,11]$} & 0.99 & 0.99 & 105 & 1925 & 0.99 & 0.99 & 106 & 1925 \\
\hline & {$[10,11,12]$} & 0.99 & 0.99 & 106 & 1926 & 1.00 & 0.99 & 106 & 1925 \\
\hline \multirow[t]{5}{*}{4} & {$[6,7,8]$} & 0.97 & 0.96 & 103 & 1986 & 0.97 & 0.97 & 105 & 1987 \\
\hline & {$[7,8,9]$} & 0.99 & 0.99 & 104 & 1987 & 0.99 & 0.99 & 106 & 1988 \\
\hline & {$[8,9,10]$} & 1.00 & 1.00 & 105 & 1988 & 1.00 & 1.00 & 104 & 1988 \\
\hline & {$[9,10,11]$} & 1.00 & 1.00 & 106 & 1990 & 1.00 & 1.00 & 106 & 1990 \\
\hline & {$[10,11,12]$} & 1.00 & 1.00 & 108 & 1990 & 1.00 & 1.00 & 108 & 1990 \\
\hline \multirow[t]{5}{*}{5} & {$[6,7,8]$} & 0.99 & 0.99 & 106 & 2242 & 0.99 & 0.99 & 104 & 2242 \\
\hline & {$[7,8,9]$} & 1.00 & 1.00 & 107 & 2244 & 1.00 & 1.00 & 106 & 2243 \\
\hline & {$[8,9,10]$} & 1.00 & 1.00 & 106 & 2245 & 1.00 & 1.00 & 107 & 2245 \\
\hline & {$[9,10,11]$} & 1.00 & 1.00 & 109 & 2245 & 1.00 & 1.00 & 109 & 2245 \\
\hline & {$[10,11,12]$} & 1.00 & 1.00 & 109 & 2247 & 1.00 & 1.00 & 110 & 2246 \\
\hline
\end{tabular}

(b) Brain amygdala tissue

\begin{tabular}{|c|c|c|c|c|c|c|c|c|c|}
\hline \multirow[t]{3}{*}{ k-mer size } & \multirow[t]{3}{*}{ Feature size } & \multicolumn{8}{|c|}{ Feature set } \\
\hline & & \multicolumn{4}{|c|}{ Forward } & \multicolumn{4}{|c|}{ Reverse } \\
\hline & & Acc & F1 & Time & Mem & Acc & F1 & Time & Mem \\
\hline \multirow[t]{5}{*}{2} & {$[6,7,8]$} & 0.58 & 0.51 & 15 & 196 & 0.59 & 0.52 & 15 & 196 \\
\hline & {$[7,8,9]$} & 0.70 & 0.63 & 13 & 196 & 0.69 & 0.63 & 13 & 196 \\
\hline & {$[8,9,10]$} & 0.76 & 0.71 & 13 & 196 & 0.77 & 0.72 & 13 & 196 \\
\hline & {$[9,10,11]$} & 0.81 & 0.76 & 13 & 196 & 0.81 & 0.76 & 13 & 196 \\
\hline & {$[10,11,12]$} & 0.84 & 0.79 & 13 & 196 & 0.84 & 0.80 & 14 & 196 \\
\hline \multirow[t]{5}{*}{3} & {$[6,7,8]$} & 0.97 & 0.96 & 13 & 199 & 0.97 & 0.96 & 13 & 199 \\
\hline & {$[7,8,9]$} & 0.99 & 0.98 & 13 & 199 & 0.99 & 0.99 & 13 & 199 \\
\hline & {$[8,9,10]$} & 1.00 & 0.99 & 13 & 199 & 1.00 & 0.99 & 13 & 199 \\
\hline & {$[9,10,11]$} & 1.00 & 1.00 & 13 & 199 & 1.00 & 1.00 & 13 & 199 \\
\hline & {$[10,11,12]$} & 1.00 & 1.00 & 13 & 199 & 1.00 & 1.00 & 13 & 199 \\
\hline \multirow[t]{5}{*}{4} & {$[6,7,8]$} & 0.99 & 0.99 & 13 & 210 & 1.00 & 0.99 & 13 & 210 \\
\hline & {$[7,8,9]$} & 1.00 & 1.00 & 14 & 210 & 1.00 & 1.00 & 13 & 210 \\
\hline & {$[8,9,10]$} & 1.00 & 1.00 & 13 & 211 & 1.00 & 1.00 & 14 & 211 \\
\hline & {$[9,10,11]$} & 1.00 & 1.00 & 13 & 211 & 1.00 & 1.00 & 13 & 211 \\
\hline & {$[10,11,12]$} & 1.00 & 1.00 & 13 & 211 & 1.00 & 1.00 & 14 & 211 \\
\hline \multirow[t]{5}{*}{5} & {$[6,7,8]$} & 1.00 & 1.00 & 13 & 256 & 1.00 & 1.00 & 14 & 256 \\
\hline & {$[7,8,9]$} & 1.00 & 1.00 & 14 & 256 & 1.00 & 1.00 & 14 & 256 \\
\hline & {$[8,9,10]$} & 1.00 & 1.00 & 14 & 256 & 1.00 & 1.00 & 14 & 256 \\
\hline & {$[9,10,11]$} & 1.00 & 1.00 & 14 & 256 & 1.00 & 1.00 & 14 & 256 \\
\hline & {$[10,11,12]$} & 1.00 & 1.00 & 14 & 270 & 1.00 & 1.00 & 14 & 256 \\
\hline
\end{tabular}


Table 2 (continued)

Total run time is reported in seconds and peak memory usage in megabytes. Accuracy indicated by Acc and F1-Score indicated by $\mathrm{F} 1$

similar run times ( $\leq 15$ seconds) and peak memory ( $\leq 270 \mathrm{MB})$ with varying k-mer and feature sizes. It is worthwhile to mention that even though this is a much smaller dataset, underfitting still occurs with a k-mer size of 2, while overfitting still occurs with k-mer sizes 4 and 5 . The fit is still optimal with a k-mer size of 3 . In contrast to the results seen in Test- 1 , the Test- 2 model overfits with feature size $[8,9,10]$. The fit is always optimal with feature size $[7,8,9]$.

As a result, we conclude that k-mer size 3 is an optimal fit for the machine learning model, whereas the optimal feature size scales with the number of genes. Both the forward and reverse feature sets in the two datasets gave similar accuracy and F1-scores which shows that using the SNPs with the highest and lowest p-values contains similar discriminatory power.

Table 3 shows the effects of varying feature sizes on the Test-3 dataset of 49 human tissues when the k-mer size is set to 3 . As expected, a smaller feature size is a good fit for tissue with fewer genes compared to a tissue with a more significant number of genes where a larger feature size is a better fit. Generally, depending on the number of genes and the purpose of the analysis, feature sizes greater than $[6,7,8]$ but less than $[9,10,11]$ is an optimal fit for the machine learning model. A similar observation was seen for the reverse feature set and is attached as Additional file 2: Table S1.

The purpose of the final experiment is different from the previous experiments, as it focuses on the ability of the expression levels of SNPs to differentiate common genes that exist across the 49 human tissues. Table 4 summarizes the results for the Test- 4 dataset comprising 8,575 genes when k-mer of size 3 is used. In this case, we conclude that feature size $[9,10,11]$ is an optimal fit for the machine learning model.

\section{Conclusions}

Our results demonstrate that our approach yields practical performance results on realworld SNP datasets. A total of 25 SNPs is sufficient to represent the highly expressed SNPs as both the forward and reverse feature sets gave similar accuracy and F1-scores. Generally, a k-mer of size 3 is an good fit while other k-mer sizes either underfit or overfit the machine learning model. On the other hand, in regards to feature sizes, depending on the number of genes and the purpose of the analysis, feature sizes $[7,8,9]$ and $[8,9$, $10]$ are typically optimal for the machine learning model. We also show that variation in the significant gene expression levels can distinguish the genes and the tissues the genes are expressed in. These distinguishing genes are potential molecular markers or therapeutic targets for disease progression. Therefore, these genes are good candidates for identifying potential SNPs associated with disease progression for future studies.

In this paper, we have (1) proposed a novel feature extraction algorithm based on feature sizes; (2) proposed an optimal k-mer of size 3 and feature size $[7,8,9]$ or $[8,9,10]$ depending on the number of genes and the application for tissue-specific genes prediction; and (3) provided a comprehensive analysis of the experimental results. We have 
Table 3 Effects of varying feature sizes on all the Test-3 dataset when k-mer size is 3 for the forward feature set

\begin{tabular}{|c|c|c|c|c|c|c|c|c|c|c|}
\hline \multirow[t]{3}{*}{ Tissue } & \multicolumn{10}{|c|}{ Feature size } \\
\hline & \multicolumn{2}{|c|}{$[6,7,8]$} & \multicolumn{2}{|c|}{$[7,8,9]$} & \multicolumn{2}{|c|}{$[8,9,10]$} & \multicolumn{2}{|c|}{$[9,10,11]$} & \multicolumn{2}{|c|}{$\begin{array}{l}{[10,11,} \\
12]\end{array}$} \\
\hline & Acc & $\mathrm{F} 1$ & Acc & F1 & Acc & $\mathrm{F} 1$ & Acc & F1 & Acc & F1 \\
\hline Adipose subcutaneous & 0.84 & 0.80 & 0.93 & 0.91 & 0.97 & 0.97 & 0.99 & 0.99 & 0.99 & 0.99 \\
\hline Adipose visceral omentum & 0.87 & 0.84 & 0.95 & 0.94 & 0.98 & 0.97 & 0.99 & 0.99 & 0.99 & 0.99 \\
\hline Adrenal gland & 0.91 & 0.89 & 0.96 & 0.95 & 0.98 & 0.98 & 0.99 & 0.99 & 1.00 & 1.00 \\
\hline Artery aorta & 0.86 & 0.83 & 0.95 & 0.93 & 0.98 & 0.97 & 0.99 & 0.98 & 0.99 & 0.99 \\
\hline Artery coronary & 0.94 & 0.92 & 0.98 & 0.97 & 0.99 & 0.99 & 1.00 & 0.99 & 1.00 & 1.00 \\
\hline Artery tibial & 0.84 & 0.81 & 0.93 & 0.92 & 0.97 & 0.96 & 0.99 & 0.98 & 0.99 & 0.99 \\
\hline Brain amygdala & 0.96 & 0.96 & 0.98 & 0.98 & 0.99 & 0.99 & 1.00 & 1.00 & 1.00 & 1.00 \\
\hline Brain anterior cingulate cortex & 0.94 & 0.93 & 0.97 & 0.97 & 0.99 & 0.99 & 1.00 & 0.99 & 1.00 & 1.00 \\
\hline Brain caudate basal ganglia & 0.92 & 0.90 & 0.97 & 0.96 & 0.99 & 0.98 & 0.99 & 0.99 & 1.00 & 1.00 \\
\hline Brain cerebellar hemisphere & 0.90 & 0.88 & 0.96 & 0.95 & 0.98 & 0.98 & 0.99 & 0.99 & 1.00 & 0.99 \\
\hline Brain cerebellum & 0.88 & 0.85 & 0.96 & 0.95 & 0.98 & 0.98 & 0.99 & 0.99 & 1.00 & 0.99 \\
\hline Brain cortex & 0.91 & 0.89 & 0.97 & 0.96 & 0.99 & 0.98 & 0.99 & 0.99 & 1.00 & 1.00 \\
\hline Brain frontal cortex & 0.93 & 0.91 & 0.97 & 0.96 & 0.99 & 0.98 & 0.99 & 0.99 & 1.00 & 1.00 \\
\hline Brain hippocampus & 0.94 & 0.93 & 0.98 & 0.98 & 0.99 & 0.99 & 1.00 & 0.99 & 1.00 & 1.00 \\
\hline Brain hypothalamus & 0.95 & 0.93 & 0.98 & 0.97 & 0.99 & 0.99 & 1.00 & 1.00 & 1.00 & 1.00 \\
\hline Brain nucleus accumbens basal ganglia & 0.92 & 0.90 & 0.97 & 0.96 & 0.99 & 0.99 & 0.99 & 0.99 & 1.00 & 1.00 \\
\hline Brain putamen basal ganglia & 0.93 & 0.91 & 0.98 & 0.97 & 0.99 & 0.99 & 1.00 & 0.99 & 1.00 & 1.00 \\
\hline Brain spinal cord cervical & 0.96 & 0.95 & 0.98 & 0.98 & 0.99 & 0.99 & 1.00 & 1.00 & 1.00 & 1.00 \\
\hline Brain substantia nigra & 0.97 & 0.96 & 0.99 & 0.98 & 0.99 & 0.99 & 1.00 & 1.00 & 1.00 & 1.00 \\
\hline Breast mammary & 0.89 & 0.86 & 0.95 & 0.94 & 0.98 & 0.98 & 0.99 & 0.99 & 1.00 & 0.99 \\
\hline Cells cultured fibroblasts & 0.84 & 0.81 & 0.93 & 0.92 & 0.97 & 0.97 & 0.99 & 0.98 & 0.99 & 0.99 \\
\hline Cells EBV transformed lymphocytes & 0.94 & 0.93 & 0.98 & 0.97 & 0.99 & 0.99 & 1.00 & 0.99 & 1.00 & 1.00 \\
\hline Colon sigmoid & 0.89 & 0.86 & 0.96 & 0.94 & 0.98 & 0.97 & 0.99 & 0.99 & 0.99 & 0.99 \\
\hline Colon transverse & 0.88 & 0.85 & 0.95 & 0.93 & 0.98 & 0.97 & 0.99 & 0.99 & 1.00 & 0.99 \\
\hline Esophagus gastroesophageal junction & 0.89 & 0.86 & 0.96 & 0.95 & 0.98 & 0.98 & 0.99 & 0.99 & 0.99 & 0.99 \\
\hline Esophagus mucosa & 0.85 & 0.82 & 0.94 & 0.92 & 0.97 & 0.97 & 0.99 & 0.98 & 0.99 & 0.99 \\
\hline Esophagus muscularis & 0.85 & 0.82 & 0.94 & 0.93 & 0.98 & 0.97 & 0.99 & 0.98 & 0.99 & 0.99 \\
\hline Heart atrial appendage & 0.88 & 0.86 & 0.95 & 0.94 & 0.98 & 0.97 & 0.99 & 0.99 & 1.00 & 0.99 \\
\hline Heart left ventricle & 0.91 & 0.88 & 0.96 & 0.95 & 0.98 & 0.98 & 0.99 & 0.99 & 1.00 & 1.00 \\
\hline Kidney cortex & 0.99 & 0.99 & 1.00 & 1.00 & 1.00 & 1.00 & 1.00 & 1.00 & 1.00 & 1.00 \\
\hline Liver & 0.94 & 0.92 & 0.97 & 0.97 & 0.99 & 0.98 & 1.00 & 1.00 & 1.00 & 1.00 \\
\hline Lung & 0.86 & 0.82 & 0.94 & 0.93 & 0.98 & 0.97 & 0.99 & 0.98 & 0.99 & 0.99 \\
\hline Minor salivary gland & 0.95 & 0.94 & 0.98 & 0.97 & 0.99 & 0.99 & 1.00 & 0.99 & 1.00 & 1.00 \\
\hline Muscle skeletal & 0.86 & 0.82 & 0.94 & 0.92 & 0.98 & 0.97 & 0.99 & 0.99 & 0.99 & 0.99 \\
\hline Nerve tibial & 0.82 & 0.78 & 0.92 & 0.90 & 0.97 & 0.96 & 0.99 & 0.98 & 0.99 & 0.99 \\
\hline Ovary & 0.94 & 0.93 & 0.98 & 0.97 & 0.99 & 0.99 & 1.00 & 1.00 & 1.00 & 1.00 \\
\hline Pancreas & 0.90 & 0.87 & 0.96 & 0.95 & 0.98 & 0.98 & 0.99 & 0.99 & 1.00 & 0.99 \\
\hline Pituitary & 0.91 & 0.88 & 0.96 & 0.95 & 0.99 & 0.98 & 0.99 & 0.99 & 1.00 & 1.00 \\
\hline Prostate & 0.93 & 0.91 & 0.97 & 0.97 & 0.99 & 0.98 & 1.00 & 0.99 & 1.00 & 1.00 \\
\hline Skin not sun exposed suprapubic & 0.84 & 0.80 & 0.93 & 0.92 & 0.97 & 0.96 & 0.99 & 0.98 & 0.99 & 0.99 \\
\hline Skin sun exposed lower leg & 0.84 & 0.80 & 0.93 & 0.91 & 0.97 & 0.96 & 0.99 & 0.98 & 0.99 & 0.99 \\
\hline Small intestine terminal ileum & 0.94 & 0.92 & 0.98 & 0.97 & 0.99 & 0.99 & 1.00 & 0.99 & 1.00 & 1.00 \\
\hline Spleen & 0.89 & 0.86 & 0.95 & 0.94 & 0.98 & 0.98 & 0.99 & 0.99 & 1.00 & 0.99 \\
\hline Stomach & 0.91 & 0.88 & 0.96 & 0.95 & 0.98 & 0.98 & 0.99 & 0.99 & 1.00 & 1.00 \\
\hline Testis & 0.83 & 0.79 & 0.93 & 0.91 & 0.97 & 0.96 & 0.99 & 0.98 & 0.99 & 0.99 \\
\hline
\end{tabular}


Table 3 (continued)

\begin{tabular}{|c|c|c|c|c|c|c|c|c|c|c|}
\hline \multirow[t]{3}{*}{ Tissue } & \multicolumn{10}{|c|}{ Feature size } \\
\hline & \multicolumn{2}{|c|}{$[6,7,8]$} & \multicolumn{2}{|c|}{$[7,8,9]$} & \multicolumn{2}{|c|}{$[8,9,10]$} & \multicolumn{2}{|c|}{$[9,10,11]$} & \multicolumn{2}{|c|}{$\begin{array}{l}{[10,11,} \\
12]\end{array}$} \\
\hline & Acc & F1 & Acc & F1 & Acc & $\mathrm{F} 1$ & Acc & F1 & Acc & $\mathrm{F} 1$ \\
\hline Thyroid & 0.82 & 0.78 & 0.92 & 0.90 & 0.97 & 0.96 & 0.98 & 0.98 & 0.99 & 0.99 \\
\hline Uterus & 0.96 & 0.95 & 0.99 & 0.98 & 0.99 & 0.99 & 1.00 & 1.00 & 1.00 & 1.00 \\
\hline Vagina & 0.97 & 0.96 & 0.99 & 0.98 & 0.99 & 0.99 & 1.00 & 1.00 & 1.00 & 1.00 \\
\hline Whole blood & 0.86 & 0.83 & 0.94 & 0.93 & 0.98 & 0.97 & 0.99 & 0.99 & 0.99 & 0.99 \\
\hline MEAN & 0.90 & 0.88 & 0.96 & 0.95 & 0.98 & 0.98 & 0.99 & 0.99 & 1.00 & 0.99 \\
\hline STDEV & 0.05 & 0.06 & 0.02 & 0.02 & 0.01 & 0.01 & 0.00 & 0.00 & 0.00 & 0.00 \\
\hline MIN & 0.82 & 0.78 & 0.92 & 0.90 & 0.97 & 0.96 & 0.98 & 0.98 & 0.99 & 0.99 \\
\hline MAX & 0.99 & 0.99 & 1.00 & 1.00 & 1.00 & 1.00 & 1.00 & 1.00 & 1.00 & 1.00 \\
\hline
\end{tabular}

Accuracy indicated by Acc and F1-Score indicated by F1

Table 4 Effects of varying feature size on a dataset that comprises of all the common genes that exist across of all the 49 human tissues

\begin{tabular}{lllll}
\hline Feature size & \multicolumn{2}{l}{ Featureset } & & \\
\cline { 2 - 3 } & \multicolumn{2}{l}{ Forward } & & Reverse \\
\cline { 2 - 4 } & Acc & F1 & Acc & F1 \\
\hline$[6,7,8]$ & 0.82 & 0.78 & 0.87 & 0.84 \\
{$[7,8,9]$} & 0.91 & 0.89 & 0.95 & 0.93 \\
{$[8,9,10]$} & 0.95 & 0.94 & 0.98 & 0.97 \\
{$[9,10,11]$} & 0.97 & 0.97 & 0.99 & 0.99 \\
{$[10,11,12]$} & 0.98 & 0.98 & 1.00 & 0.99 \\
\hline
\end{tabular}

The k-mer size is set to 3 . Accuracy indicated by Acc and F1-Score indicated by F1

shown that patterns learned from SNP expression levels with the highest and lowest p-values contain similar discriminatory power.

\section{Abbreviations}

SNPs: Single nucleotide polymorphisms; MNB: Multinomial naive Bayes; DNA: Deoxyribonucleic acid; NLP: Natural language processing; GTEx: Genotype-tissue expression.

\section{Supplementary Information}

The online version contains supplementary material available at https://doi.org/10.1186/s40537-021-00497-9.

Additional file 1. Code S1.

Additional file 2: Table S1. Effects of varying feature sizes on all the Test-3 dataset when $\mathrm{k}$-mer size is 3 for the reverse feature set. Accuracy indicated by Acc and F1-Score indicated by F1.

\section{Acknowledgements}

The Genotype-Tissue Expression (GTEx) Project was supported by the Common Fund of the Office of the Director of the National Institutes of Health, and by NCI, NHGRI, NHLBI, NIDA, NIMH, and NINDS. The data used for the analyses described in this manuscript were obtained from: Single-Tissue cis-QTL Data, GTEx_Analysis_V8_eQTL.tar the GTEx Portal on 15/02/2021. The authors thank three anonymous reviewers for their comments that materially improved the manuscript. JD dedicates this manuscript to the memory of Buddy. 


\section{Authors' contributions}

JD conceived the project, designed and implemented the algorithm, designed and ran the experiments, and wrote the manuscript. JD and JW interpreted the results. JW provided biological insights, reviewed and edited the manuscript. Both authors read and approved the final manuscript.

\section{Funding}

Not applicable.

\section{Availability of data and materials}

The datasets analyzed during the current study are available from the Single-Tissue cis-QTL Data, GTEx_Analysis_v8_ eQTL.tar (https://www.gtexportal.org/home/datasets).

\section{Declarations}

Ethics approval and consent to participate

Not applicable.

\section{Consent for publication}

Not applicable.

\section{Competing interests}

The authors declare that they have no competing interests.

\section{Author details}

${ }^{1}$ School of Information Technology, Monash University Malaysia, Subang Jaya 47500, Malaysia. ${ }^{2}$ IBM Research-Australia, Southbank, VIC 3006, Australia.

Received: 27 April 2021 Accepted: 30 July 2021

Published online: 17 August 2021

\section{References}

1. Consortium IHGS. Initial sequencing and analysis of the human genome. Nature. 2001;409:860-921.

2. Project TIH. The international hapmap project consortium. Nature. 2003:426:789-96.

3. Quezada H, Guzmán-Ortiz A, Díaz-Sánchez H, Valle-Rios R, Aguirre-Hernández J. Omics-based biomarkers: current status and potential use in the clinic. Boletín Médico del Hospital Infantil de México. 2017:74(3):219-26.

4. Kathiresan S, Melander O, Anevski D, Guiducci C, Burtt N, Roos C, Hirschhorn JN, Berglund G, Hedblad B, Groop L, Altshuler DM, Newton-Cheh C, Orho-Melander M. Polymorphisms associated with cholesterol and risk of cardiovascular events. N Engl J Med. 2008;358(12):1240-9.

5. Miranda D, Romano-Silva MA, De Marco L. Single nucleotide polymorphisms (snps) and the search for obesityrelated genes. Arquivos Brasileiros de Endocrinologia Metabologia. 2008;52(4):577-8.

6. Bertout J, Patel S, Simon M. The impact of o2 availability on human cancer. Nat Rev Cancer. 2008;8(12):967-75.

7. Alkorta-Aranburu G, Beall CM, Witonsky DB, Gebremedhin A, Pritchard JK, Rienzo AD. The genetic architecture of adaptations to high altitude in Ethiopia. PLoS Genet. 2012;8(12):1003110.

8. Christiansen M, Larsen S, Nyegaard M, Neergaard-Petersen S, Ajjan R, Würtz M, Grove EL, Hvas A-M, Jensen HK, Kristensen S. Coronary artery disease-associated genetic variants and biomarkers of inflammation. PLoS ONE. 2017;12(7):0180365

9. Rashkin SR, Chua KC, Ho C, Mulkey F, Jiang C, Mushiroda T, Kubo M, Friedman PN, Rugo HS, McLeod HL, Ratain MJ, Castillos F, Naughton M, Overmoyer B, Toppmeyer D, Witte JS, Owzar K, Kroetz DL. A pharmacogenetic prediction model of progression-free survival in breast cancer using genome-wide genotyping data from calgb 40502 (alliance). Clin Pharmacol Ther. 2019;105(3):738-45.

10. Wei Z, Wang W, Bradfield J, Li J, Cardinale C, Frackelton E, Kim C, Mentch F, Van Steen K, Visscher PM, Baldassano $\mathrm{RN}$, Hakonarson $\mathrm{H}$, the International IBD Genetics Consortium. Large sample size, wide variant spectrum, and advanced machine-learning technique boost risk prediction for inflammatory bowel disease. Am J Hum Genet. 2013;92(6):1008-12.

11. Montanez CAC, Fergus P, Montanez AC, Hussain A, Al-Jumeily D, Chalmers C. Deep learning classification of polygenic obesity using genome wide association study snps. In: International Joint Conference on Neural Networks (IJCNN), pp. 1-8; 2018.

12. Uddin S, Khan A, Hossain ME, Moni MA. Comparing different supervised machine learning algorithms for disease prediction. BMC Med Inf Decis Making. 2019;19:281.

13. Hsiao L, Dangond F, Yoshida T, Hong R, Jensen R, Misra J, Dillon W, Lee KF, Clark KE, Haverty P, Weng Z, Mutter GL, Frosch MP, MacDonald ME, Milford EL, Crum CP, Bueno R, Pratt RE, Mahadevappa M, Warrington JA, Stephanopoulos G, Stephanopoulos G, Gullans S. A compendium of gene expression in normal human tissues. Physiol Genomics. 2001;7(2):97-104

14. NIH National Human Genome Research Institute. The Genotype-Tissue Expression Project (GTEx). https://www. genome.gov/27549432/gtex-surgical-donors. Accessed 20 Oct 2020

15. NIH National Institutes of Health Office of Strategic Coordination - The Common Fund. Genotype-Tissue Expression. https://commonfund.nih.gov/gtex. Accessed 26 Apr 2021

16. Sonawane AR, Platig J, Fagny M, Chen C-Y, Paulson JN, Lopes-Ramos CM, DeMeo DL, Quackenbush J, Glass K, Kuijjer ML. Understanding tissue-specific gene regulation. Cell Rep. 2017;21(4):1077-88 
17. NIH National Library of Medicine National Center for Biotechnology Information. ClinVar Genomic variation as it relates to human health. https://www.ncbi.nlm.nih.gov/clinvar/variation/1062/. Accessed 7 July 2021

18. Haruechaiyasak C, Kongyoung S, Dailey M. A comparative study on Thai word segmentation approaches. International Conference on Electrical Engineering/Electronics, Computer, Telecommunications and Information Technology. 2008;1:125-8.

19. Shao Y, Hardmeier C, Nivre J. Universal word segmentation: implementation and interpretation. Trans Assoc Comput Linguist. 2018;6:421-35.

20. Clercq GD. Deep learning for classification of dna functional sequences. Ghent University; 2019. Master's thesis.

21. Brownlee J. How to Encode Text Data for Machine Learning with scikit-learn. https://machinelearningmastery.com/ prepare-text-data-machine-learning-scikit-learn/. Accessed 10 Oct 2020

22. Lebret RP. Word embeddings for natural language processing. PhD thesis, Ecole Polytechnique Fédérale de Lausanne. 2016.

23. Kuksa P, Pavlovic V. Efficient alignment-free dna barcode analytics. BMC Bioinform. 2009;10:9.

24. Fiannaca A, La Rosa M, Rizzo R, Urso A. A k-mer-based barcode dna classification methodology based on spectral representation and a neural gas network. Artifi Intell Med. 2015;64(3):173-84.

25. Rizzo R, Fiannaca A, Rosa ML, Urso A. A deep learning approach to DNA sequence classification. In: Angelini C, Rancoita P, Rovetta, S. (eds.) Computational Intelligence Methods for Bioinformatics and Biostatistics (CIBB). Lecture Notes in Computer Science, vol. 9874, pp. 129-40. Springer, 2016.

26. Nguyen NG, Tran VA, Ngo DL, Phan D, Lumbanraja FR, Faisal MR, Abapihi1 B, Kubo M, Satou K. Dna sequence classification by convolutional neural network. J Biomed Sci Eng. 2016;9:280-6.

27. Pokholok DK, Harbison CT, Levine S, Cole M, Hannett NM, Lee TI, Bell GW, Walker K, Rolfe PA, Herbolsheimer E, Zeitlinger J, Lewitter F, Gifford DK, Young RA. Genome-wide map of nucleosome acetylation and methylation in yeast. Cell. 2005;122(4):517-27.

28. Mathé C, Sagot M, Schiex T, Rouzé P. Current methods of gene prediction, their strengths and weaknesses. Nucleic Acids Res. 2002;30(19):4103-17.

29. Fickett J, Tung C-S. Assessment of protein coding measures. Nucleic Acids Res. 1992;20(24):6441-50

30. GTExPortal: GTExPortal. http://gtexportal.org/home/. Accessed 20 Feb 2021

31. Domingos P, Pazzani M. On the optimality of the simple Bayesian classifier under zero-one loss. Mach Learn. 1997:29:103-30

32. Ismail M, Hassan N, Bafjaish SS. Comparative analysis of Naive Bayesian techniques in health-related for classification task. J Soft Comput Data Mining. 2020;1 (2):1-10.

33. Ashari A, Paryudi I, Tjoa AM. Performance comparison between naïve bayes, decision tree and k-nearest neighbor in searching alternative design in an energy simulation tool. International Journal of Advanced Computer Science and Applications. 2013;4(11).

34. Hatzivassiloglou V, Duboué P, Rzhetsky A. Disambiguating proteins, genes, and rna in text: a machine learning approach. Bioinformatics. 2001;17(Suppl 1):97-106.

\section{Publisher's Note}

Springer Nature remains neutral with regard to jurisdictional claims in published maps and institutional affiliations.

\section{Submit your manuscript to a SpringerOpen ${ }^{\circ}$ journal and benefit from:}

- Convenient online submission

Rigorous peer review

Open access: articles freely available online

- High visibility within the field

- Retaining the copyright to your article

Submit your next manuscript at $\gg$ springeropen.com 